\title{
Hernia hiatal gigante con estómago intratorácico: Reporte de un caso y revisión de la literatura
}

\author{
Dres. José A. de Grazia $K^{(1,2)}$, Marcelo Godoy $Z^{(1,2)}$, Ítalo Cavallo $B^{(1,2)}$, Claudio Cortés $A^{(1)}$.
}

1. Centro de Imagenología, Hospital Clínico Universidad de Chile, Santiago - Chile.

2. Médico Becario de Radiología.

\section{Giant hiatal hernia with intrathoracic stomach: A case report and literature review}

\begin{abstract}
Giant hiatal hernia (GHH) with intrathoracic stomach is a rare condition. It is a type 3 or mixed hernia (with sliding and paraesophageal component) with more than $30 \%$ of the stomach protruding into the chest cavity. These hernias are usually associated with gastric rotation, mostly with organoaxial twist. They have nonspecific clinical manifestations, which include vomiting, and chest or epigastric pain. Complications such as volvulus, perforation, or gastric obstruction constitute a medical emergency. In many cases, these hernias can be detected incidentally on a chest radiograph. Imaging studies, mainly multidetector CT and upper gastro-intestinal series represent vital tools for diagnosis and anatomical characterization of these lesions. The treatment of GHH requires an open or laparoscopic surgical approach. We report the case of a patient evaluated in our department who was diagnosed with giant hiatal hernia with intrathoracic stomach and organoaxial gastric rotation.
\end{abstract}

Keywords: Computed x-ray tomography, Gastric volvulus, Hiatal hernia.

Resumen: La hernia hiatal gigante (HHG) con estómago intratorácico es una enfermedad poco frecuente. Corresponde a una hernia tipo 3 o mixta (componente por deslizamiento y paraesofágico) que contiene más del 30\% del estómago a nivel del tórax. Es común que estas hernias se asocien a rotación gástrica, preferentemente del tipo órgano-axial. Sus manifestaciones clínicas son inespecíficas e incluyen vómitos y dolor torácico o epigástrico. Pueden presentar complicaciones como vólvulo, perforación u obstrucción gástrica, constituyendo una emergencia médica. En muchos casos pueden ser detectadas en forma incidental en una radiografía de tórax. El estudio dirigido con imágenes, particularmente tomografía computada multidetector y estudio baritado esófago-estómago-duodeno, resultan fundamentales para el diagnóstico y caracterización de la anatomía de estas lesiones. El tratamiento es quirúrgico, sea por cirugía abierta o laparoscópica. Presentamos el caso de una paciente evaluada en nuestro servicio, en quien se realizó el diagnóstico de HHG con estómago intratorácico, con rotación gástrica órgano-axial.

Palabras clave: Hernia hiatal, Tomografía computada, Vólvulo gástrico.

de Grazia, et al. Hernia hiatal gigante con estómago intratorácico: Reporte de un caso y revisión de la literatura. Rev Chil Radiol 2012; 18(4): 179-183.

Correspondencia: Dr. José A.de Grazia K. / ja.degrazia@gmail.com

Trabajo recibido el 01 de diciembre de 2011, aceptado para publicación el 06 de marzo de 2012.

\section{Introducción}

La hernia hiatal gigante $(\mathrm{HHG})$ con estómago intratorácico es una enfermedad poco frecuente. Durante el último tiempo hemos visto casos con mayor frecuencia en pacientes añosos y obesos. Habitualmente estas hernias se asocian a rotación gástrica órgano-axial, y pueden presentar complicaciones como vólvulo, perforación u obstrucción gástrica. El estudio con imágenes, particularmente tomografía computada multidetector y estudio baritado esófagoestómago-duodeno, resultan fundamentales para el diagnóstico y caracterización de estas lesiones. El tratamiento es quirúrgico, sea por cirugía abierta o laparoscópica.

\section{Caso clínico:}

Paciente mujer, de 72 años, con antecedente de enfermedad pulmonar obstructiva crónica (EPOC) 
en tratamiento. Presenta vómito y dolor torácico postprandial intermitente desde hace años. Durante la última semana aumenta la frecuencia de los vómitos. Consulta en el Servicio de Urgencia de nuestro hospital.

Se cuenta con radiografía de tórax de hace 2 años, que muestra una imagen redondeada, con nivel hidroaéreo, sobreproyectada a la silueta cardíaca; en la proyección lateral se comprueba la localización retrocardíaca de esta lesión (Figura 1).

Se continúa el estudio con tomografía computada de tórax y abdomen con contraste. Se evidencia una gran hernia hiatal, con la unión esófago-gástrica deslizada hacia el tórax y con el fondo, parte del cuerpo y el antro gástrico también situados en posición intratorácica. Lo anterior, configura una hernia hiatal tipo 3 o mixta. El estómago se encuentra rotado en forma órgano-axial. La porción del cuerpo gástrico que se ubica a nivel intraabdominal, sobredistendida, provoca compresión de la región antro-pilórica contra el pilar diafragmático derecho. La pared gástrica realza adecuadamente con el medio de contraste, no presenta neumatosis ni líquido circundante (Figuras 2 y 3$)$.

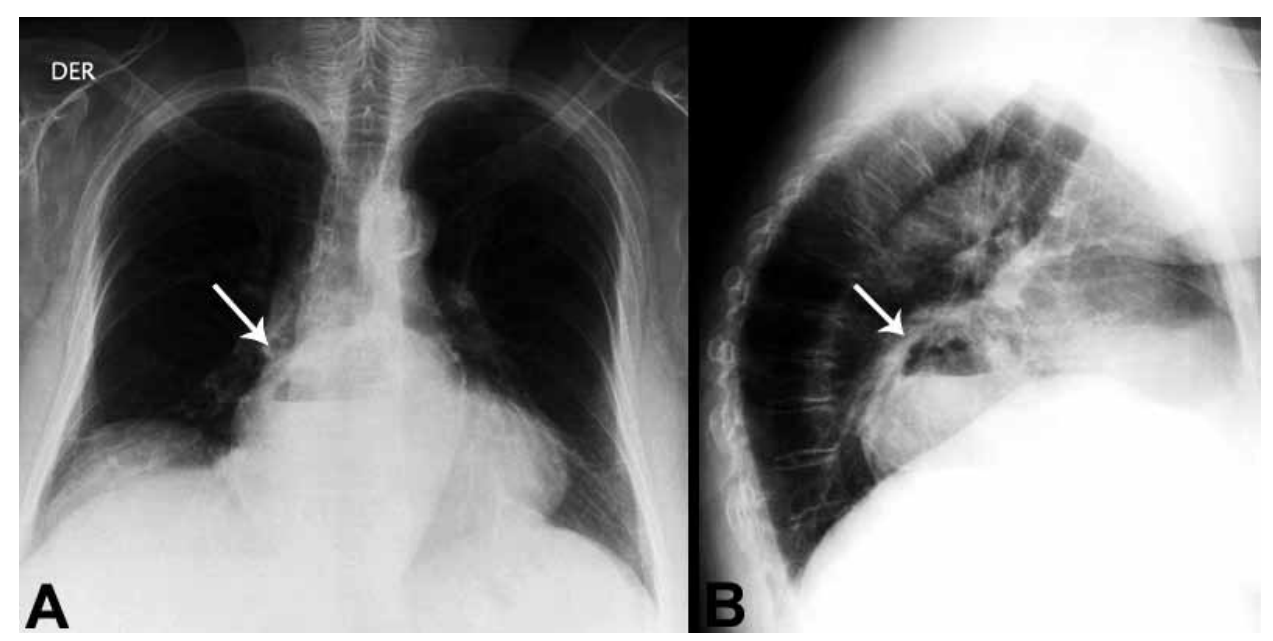

Figura 1. Radiografía de tórax, proyección póstero-anterior y lateral, en paciente con hernia hiatal gigante con estómago intratorácico.

(A) proyección póstero-anterior: se observa, en el mediastino, un nivel hidroaéreo sobreproyectado a la silueta cardiaca (flecha blanca); (B) proyección lateral: se comprueba la localización retrocardiaca, en mediastino posterior, de la lesión (flecha blanca).

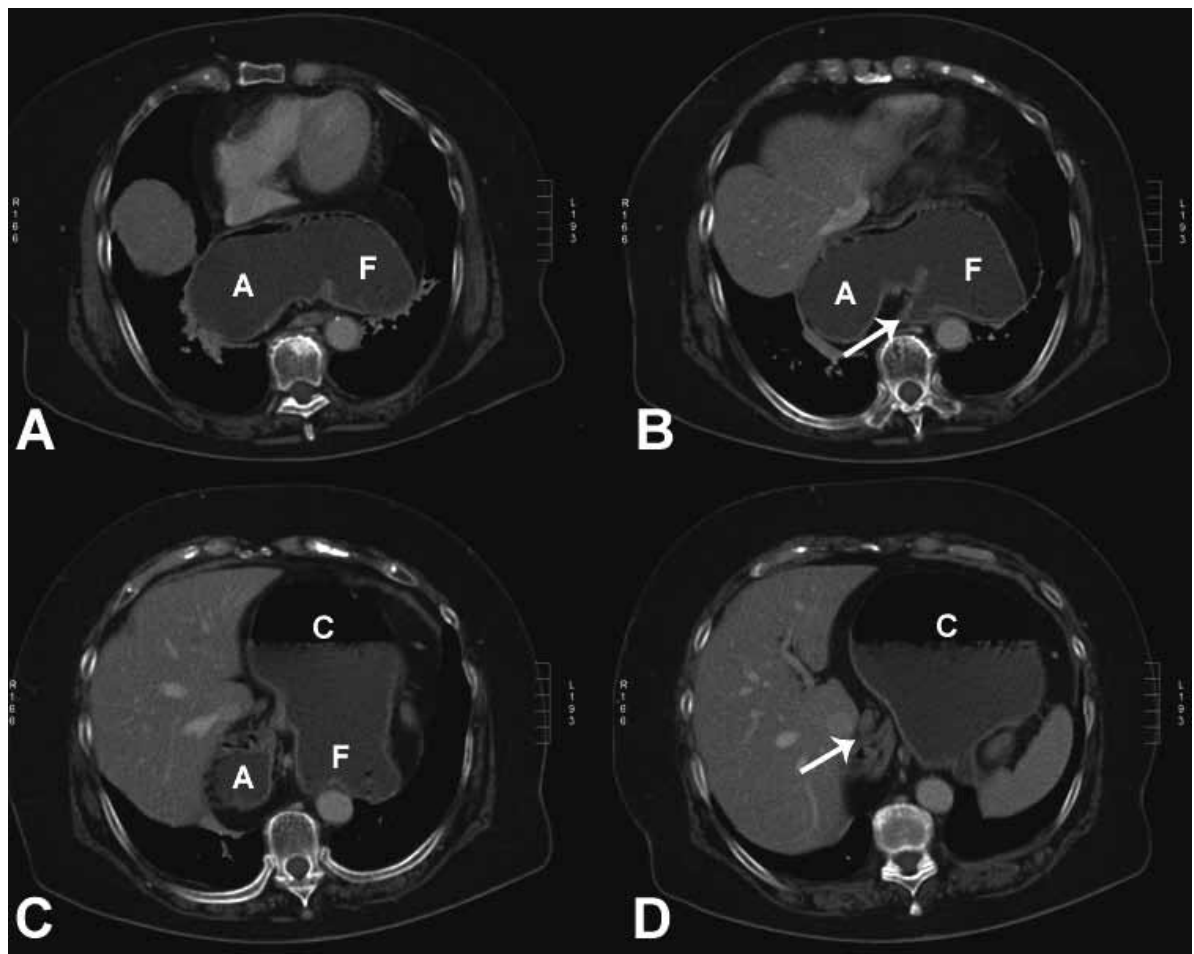

Figura 2. Tomografía computada de tórax y abdomen con contraste, cortes axiales, en paciente con hernia hiatal gigante con estómago intratorácico.

(A), (B), (C) y (D) cortes axiales, ordenados en sentido céfalocaudal: el fondo (letra F) y antro (letra $A)$ se sitúan en el tórax, el cuerpo (letra $C$ ) se sitúa parcialmente en el tórax. La unión esófago-gástrica se encuentra ascendida hacia el tórax (flecha en la imagen B). La región antropilórica está comprimida contra el pilar diafragmático derecho por el cuerpo gástrico sobredistendido (flecha en la imagen D). 
Para caracterizar la anatomía de la lesión, se realiza un estudio baritado esófago-estómago-duodeno, en el cual se observa el estómago herniado hacia el tórax, dispuesto de manera arciforme, similar a una letra griega Omega. No se evidencia contenido de retención alimentaria y el paso de contenido a través del píloro, el cual es intraabdominal, es expedito (Figura 4).

En resumen, los hallazgos radiológicos permiten realizar el diagnóstico de HHG con estómago intratorácico. Esta hernia es del tipo 3 o mixta, pues presenta un componente por deslizamiento (ascenso de la unión esófago-gástrica), y un componente paraesofágico (ascenso del fondo y antro gástrico más hacia cefálico que la unión esófago-gástrica). El estómago posee una rotación órgano-axial, ubicándose la curvatura mayor superior a la curvatura menor. La tomografía computada demuestra que no hay compromiso isquémico ni perforación del órgano.

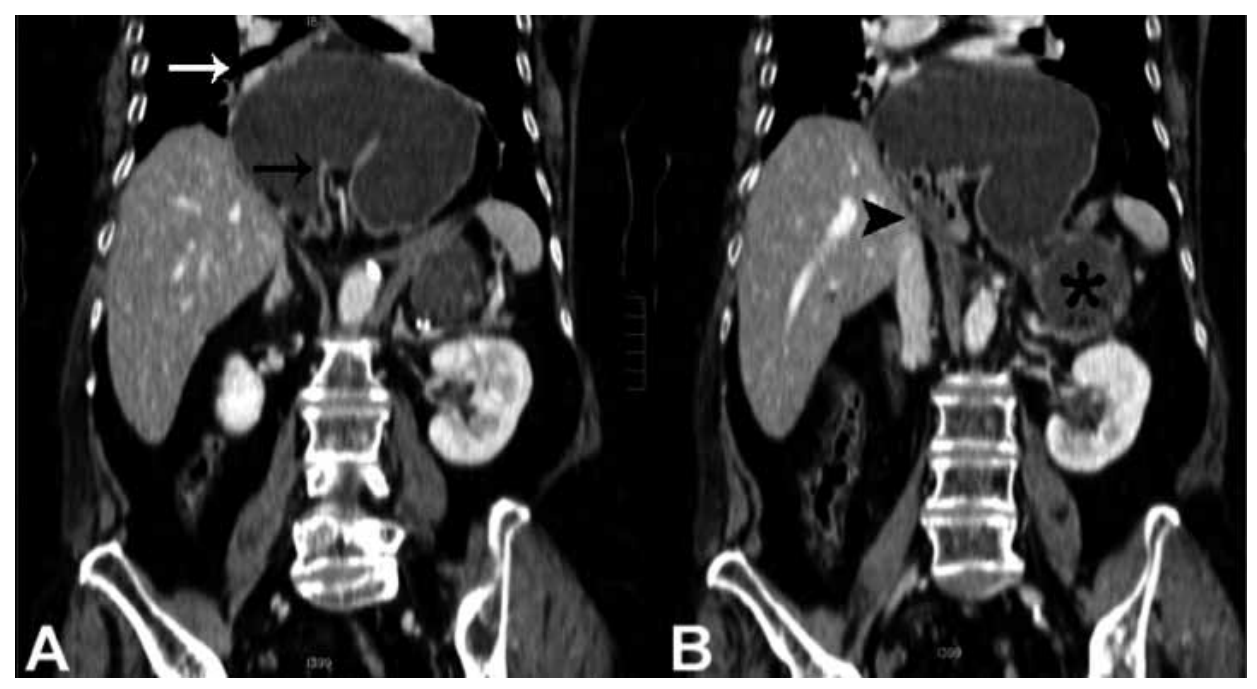

Figura 3. Tomografía computada de tórax y abdomen con contraste, reconstrucciones coronales, en paciente con hernia hiatal gigante con estómago intratorácico.

(A) reconstrucción coronal: la curvatura mayor del estómago (flecha blanca) se sitúa superior a la curvatura menor (flecha negra), constituyendo una rotación gástrica órgano-axial intratorácica; (B) reconstrucción coronal: la región antro-pilórica es comprimida contra el pilar diafragmático derecho (punta de flecha) por el cuerpo gástrico, parte del cuerpo del estómago se sitúa aún en el abdomen (asterisco).

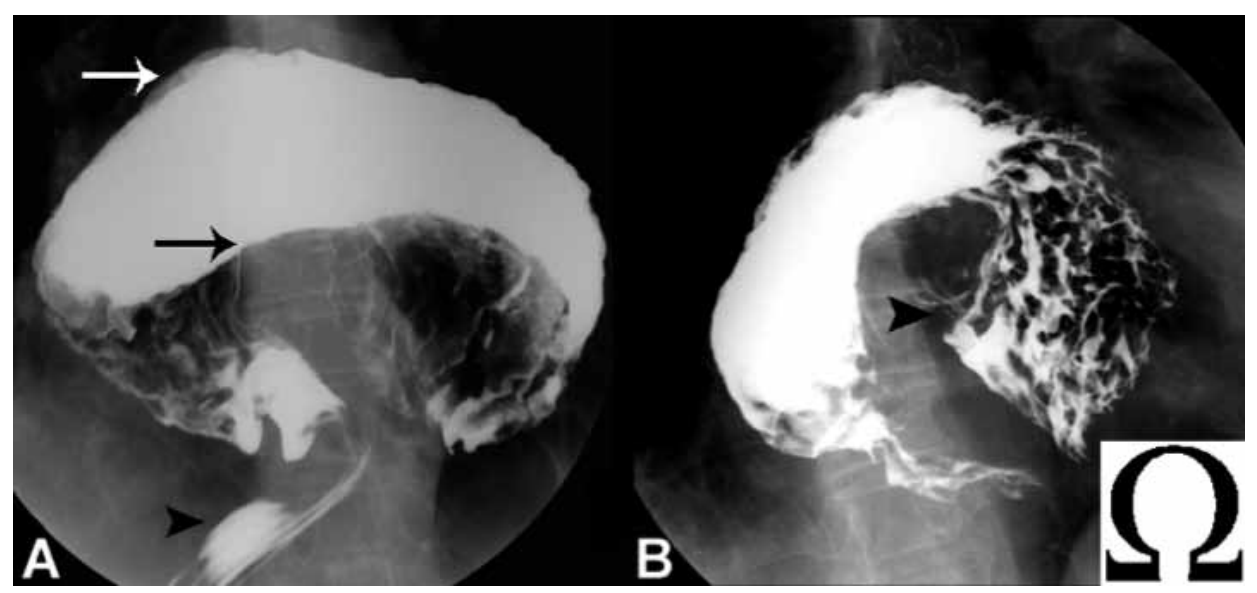

Figura 4. Estudio baritado de esófago-estómago-duodeno, imágenes del estómago, en paciente con hernia hiatal gigante con estómago intratorácico.

(A) visión frontal del estómago: la curvatura mayor del estómago (flecha blanca) se sitúa superior a la curvatura menor (flecha negra), constituyendo una rotación gástrica órgano-axial, el estómago incurvado adopta una disposición en letra griega Omega, la porción distal del antro gástrico (presenta pliegues longitudinales) se sitúa intraabdominal (punta de flecha); (B) visión oblicua del estómago: la unión esófago-gástrica se encuentra ascendida, situada en el tórax (punta de flecha), no hay retención de contenido en esófago. En la esquina inferior derecha de la imagen se ilustra la letra griega Omega, ocupada para describir la disposición del estómago. 
Durante la hospitalización, a la paciente se le pesquisa además una fibrilación auricular. Considerando este hallazgo y el antecedente de (EPOC), se decide tratamiento conservador. La paciente evoluciona favorablemente con terapia médica (inhibidores bomba de protones y proquinéticos). Debido a la mejoría sintomática, y al carácter benigno de la enfermedad, se decide mantener una conducta expectante y dar de alta a la paciente, con control.

\section{Discusión}

Existen tres tipos de hernias diafragmáticas no traumáticas. La más común es la hernia hiatal, correspondiente a la herniación del estómago, y en algunos casos otras vísceras abdominales, hacia el tórax a través del hiato esofágico ${ }^{(1,2)}$. Con menor frecuencia se presentan dos tipos de hernias congénitas: la hernia de Bochdalek (defecto en sitio del canal pleuro-peritoneal embrionario, más común al lado izquierdo) y la hernia de Morgagni (defecto del septo transverso ántero-medial, de localización retroesternal paracardiaca, más común al lado derecho) $)^{(1,2)}$.

Habitualmente las hernias hiatales contienen sólo la porción proximal del estómago, pero en ocasiones el estómago completo puede herniarse hacia el tórax ${ }^{(3,4)}$. En estos casos, el estómago asume una posición invertida, con la curvatura mayor superior a la curvatura menor. Simultáneamente, el fondo y parte del cuerpo gástrico pueden retornar, a través del hiato esofágico, a su posición normal infradiafragmática, dejando sólo a la porción distal del estómago en el tórax ${ }^{(3)}$.

En la literatura, las hernias hiatales están clasificadas en 4 categorías ${ }^{(4,5)}$ :

> Tipo 1: Corresponde a la hernia hiatal por deslizamiento. La unión esófago-gástrica está desplazada hacia el tórax, debido a debilidad y elongación de la membrana frénico-esofágica. Constituye el $95 \%$ de todas las hernias hiatales.

$>$ Tipo 2: Corresponde a la hernia hiatal paraesofágica. Existe un defecto focal, generalmente en el aspecto antero-lateral de la membrana frénicoesofágica. El cardias y la unión esófago-gástrica permanecen por debajo del diafragma. El fondo gástrico usualmente encabeza la herniación, pero todo el estómago puede migrar hacia cefálico, constituyendo un estómago intratorácico.

$>$ Tipo 3: Es una hernia hiatal mixta, con componente por deslizamiento y paraesofágico. Habitualmente es una hernia grande, asociada a rotación gástrica.

$>$ Tipo 4: Es una hernia tipo 3, que además posee herniación de otras estructuras abdominales, como colon, omento, intestino delgado o hígado. Una hernia hiatal gigante es aquella que incluye al menos el $30 \%$ del estómago en el tórax ${ }^{(6)}$. La mayoría de las veces, estas hernias corresponden a una hernia hiatal tipo 3, con componente por deslizamiento y paraesofágico(6).
A medida que el estómago se hernia hacia el tórax, éste va rotando. Más de dos tercios de las rotaciones gástricas se encuentran asociadas a hernias diafragmáticas ${ }^{(7-9)}$. La rotación más común es la rotación anterior órgano-axial, donde el estómago rota a lo largo de su eje longitudinal, con movimiento hacia anterior y luego hacia cefálico de la curvatura mayor ${ }^{(4,7,10)}$. El estómago también puede rotar en forma mesenterio-axial, es decir, en un eje perpendicular a su eje longitudinal, con movimiento hacia anterior y luego hacia la izquierda del antro ${ }^{(4,8,10)}$. El término "vólvulo gástrico" se reserva para aquellos casos en que la rotación anormal determina estrangulación y obstrucción del órgano ${ }^{(4)}$. A pesar de esto, en la literatura éste término es usado muchas veces inadecuadamente como sinónimo de "rotación gástrica".

Las manifestaciones clínicas de una hernia diafragmática son variadas. Pacientes con una hernia pequeña son usualmente asintomáticos. Las hernias tipo 1 muchas veces se asocian a incompetencia del esfínter esofágico inferior, con reflujo gastro-esofágico patológico ${ }^{(4)}$. En el caso de HHG con estómago intratorácico, la retención de alimentos y aire en el estómago herniado causa distensión del órgano, con aparición de vómitos y dolor torácico(4). En caso de hernias complicadas, con un vólvulo gástrico agudo, los síntomas incluyen dolor torácico o epigástrico de aparición súbita, náuseas y arcadas con incapacidad de vomitar y la imposibilidad de pasar una sonda naso-gástrica (triada de Borchardt) ${ }^{(4,8)}$, también pueden presentar hematemesis ${ }^{(8,9)}$. En caso de gangrena y perforación del estómago herniado, se pueden desarrollar complicaciones como mediastinitis o peritonitis.

Respecto al estudio por imágenes, las hernias hiatales gigantes con estómago intratorácico pueden manifestarse como masas mediastínicas incidentales en la radiografía de tórax, donde se ven proyectadas por detrás del corazón, en el mediastino posterior, muchas veces con un nivel hidroaéreo ${ }^{(1,4)}$. Con el uso de tomografía computada multidetector, en particular con reconstrucciones multiplanares, es posible caracterizar con detalle la anatomía de la hernia y definir de qué tipo se trata, establecer si existe rotación gástrica y de qué tipo es ésta, evaluar la herniación de otras estructuras abdominales además del estómago y, por último, establecer la presencia de complicaciones como vólvulo o perforación gástrica ${ }^{(1,4,5)}$. El estudio baritado esófago-estómago-duodeno es fundamental para caracterizar la hernia en los casos en que se planea reparación quirúrgica, siendo el examen de elección para el diagnóstico en muchos casos ${ }^{(4,5,8,9)}$; entrega información detallada respecto a la anatomía, y es el mejor estudio para determinar el tamaño de la hernia y la posición de la unión esófago-gástrica. 
La endoscopía digestiva alta juega un rol menor en el diagnóstico de hernia hiatal ${ }^{(5)}$. Su mayor utilidad radica en confirmar obstrucción gástrica y descartar lesiones mucosas concomitantes que puedan sugerir isquemia.

El tratamiento de los pacientes sintomáticos con hernia hiatal gigante con estómago intratorácico es quirúrgico. En caso de complicaciones, como vólvulo, obstrucción o perforación, la cirugía debe ser de urgencia. El abordaje quirúrgico puede ser a través del tórax o el abdomen ${ }^{(4)}$. La cirugía laparoscópica puede ser usada tanto para hernias con rotación gástrica órgano-axial como mesenterio-axial ${ }^{(12,13)}$. Dentro de las ventajas de la cirugía laparoscópica en estos pacientes, se cuenta tiempo operatorio corto, buen control de síntomas y bajas tasas de recurrencia ${ }^{(13)}$.

El caso presentado es característico de HHG con estómago intratorácico. La evolución de la paciente fue satisfactoria, a pesar de no realizarse tratamiento quirúrgico, lo cual evidencia que no existía una complicación de la hernia, como un vólvulo o una perforación gástrica. Respecto a las imágenes, la radiografía de tórax da la pista del diagnóstico, al visualizar una imagen redondeada con nivel hidroaéreo en el mediastino posterior. La tomografía computada de tórax y abdomen con contraste y el estudio baritado esófago-estómagoduodeno permiten caracterizar la anatomía de la hernia y de la rotación gástrica existente, además de descartar la presencia de otras estructuras abdominales herniadas hacia el tórax y la existencia de complicaciones como vólvulo, perforación u obstrucción gástrica. De esta manera, se llega al diagnóstico de hernia hiatal gigante con estómago intratorácico, correspondiente a una hernia tipo 3 o mixta, con rotación gástrica órgano-axial.

\section{Bibliografía}

1. Klein JS. Pleura, chest wall, diaphragm, and misceIlaneous chest disorders. In: Brant WE, Helms CA, editors. Fundamentals of Diagnostic Radiology. 3rd Ed. Lippincott Williams \& Wilkins; 2007. Chapter 19.

2. Eren S, Çiris F. Diaphragmatic hernia: diagnostic approaches with review of the literature. Eur J Radiol 2005; 54(3): 448-459.

3. Halpert RD. Esophagus and gastroesophageal junction. In: Halpert RD, editor. Gastrointestinal Imaging, The Requisits. 3rd Ed. Mosby - Elsevier; 2006. Chapter 1.

4. Abbara S, Kalan MM, Lewicki AM. Intrathoracic stomach revisited. AJR Am J Roentgenol 2003; 181(2): 403-414.

5. Kahrilas PJ, Kim HC, Pandolfino JE. Approaches to the diagnosis and grading of hiatal hernia. Best Pract Res Clin Gastroenterol 2008; 22(4): 601-616.

6. Mitiek MO, Andrade RS. Giant hiatal hernia. Ann Thorac Surg. 2010; 89(6): S2168-2173.

7. Kontorinis N, Waters TE, Zimmerman M, Kaard A. Images of interest. Gastrointestinal: gastric volvulus. J Gastroenterol Hepatol 2001; 16(2): 227.

8. Lee TC, Liu KL, Lin MT, Wang HP. Unusual cause of emesis in an octogenarian: organoaxial gastric volvulus associated with paraesophageal diaphragmatic hernia. J Am Geriatr Soc. 2006; 54(3): 555-557.

9. Al-Balas H, Hani MB, Omari HZ. Radiological features of acute gastric volvulus in adult patients. Clin Imaging 2010; 34(5): 344-347.

10. Chau B, Dufel S. Gastric volvulus. Emerg Med J 2007; 24: 446-447.

11. Chiu CC, Wang W, Wei PL, Lee WJ. Giant diaphragmatic hernia with intrathoracic gastric volvulus. Endoscopy. 2006; 38 Suppl 2: E52-53.

12. Inaba K, Sakurai Y, Isogaki J, Komori Y, Uyama I. Laparoscopic repair of hiatal hernia with mesenterioaxial volvulus of the stomach. World J Gastroenterol. 2011; 17(15): 2054-2057.

13. Wiechmann RJ, Ferguson MK, Naunheim KS, McKesey P, Hazelrigg SJ, Santucci TS, et al. Laparoscopic management of giant paraesophageal herniation. Ann Thorac Surg 2001; 71(4): 1080-1086; discussion 1086-1087. 